\title{
Primitive elements of free Lie $p$-algebras
}

\author{
G. Rakviashvili
}

Ilia State University, Tbilisi, Georgia

E-mail: giorgi.rakviashvili@iliauni.edu.ge

\begin{abstract}
Let $L$ be a finitely generated free Lie $p$-algebra and $\langle a\rangle$ an ideal generated by $a \in L$. It is proved that $L /\langle a\rangle$ is free if and only if $\langle a\rangle$ is primitive (i.e. $a$ belongs to some set of free generators of $L)$. Earlier analogues theorems were proved for some objects, for example, for groups, Lie algebras, free algebras and so on.
\end{abstract}

2010 Mathematics Subject Classification. 17B99.

Keywords. Lie $p$-algebras, primitive elements.

Introduction. It is known (1930, [4]), that if $F$ is a finitely generated free group and $a \in F$ then $\mathrm{a}$ is a primitive element (i.e. $a$ belongs to some set of free generators of $F$ ) if and only if $F /\langle a\rangle$ is a free group ( $\langle a\rangle$ denotes a normal subgroup of $F$ generated by a). Later similar theorems were proved for Lie algebras $(1970,[2])$, free algebras, free commutative algebras and free anticommutative algebras (2001, [6]). Mikhalev, Shpilrain and Umirbaev in (2004, [7]) conjectured that analogous theorem for Lie $p$-algebras is also true. In [8] the author proved Freiheitssatz for Lie $p$-algebras but with its help as it seems is impossible to prove the foresaid theorem. In this paper, we prove a theorem about primitive elements of free Lie $p$-algebras in the same manner as in (1970, [2]) using Bokuts result from [1]. Some results of our article were announced in [9].

Let $k$ be a field of characteristic $p>0, p \neq 2$, let $F=k\langle X\rangle$ be a free associative algebra without identity with $X=\left\{x_{1}, x_{2}, \ldots, x_{n}\right\}$ as a set of free generators. We will assume that $x_{i}<x_{j} \Leftrightarrow i>j$ and if $w_{1}$ and $w_{2}$ are words from $k\langle X\rangle$ then $w_{1}<w_{2}$ either degw $w_{1}<$ deg $w_{2}$ or degw $w_{1}=d e g w_{2}$ and $w_{1}<w_{2}$ lexicographically.

For $f \in F=k\langle X\rangle$, let $\bar{f}$ denote a leading word of $F$ with nonzero coefficient. We assume that the coefficient of $\bar{f}$ is equal to one. It is clear that $\bar{f} \bar{g}=\overline{f g}$.

Let $L_{p}\langle X\rangle$ denotes a free Lie $p$-algebra over $k$ with $X$ as a set of free generators. A set $Y \subset L_{p}\langle X\rangle$ is called p-independent [2] if $Y$ is a set of free generators of Lie $p$-subalgebra of $L_{p}\langle X\rangle$ generated by $Y$ (recall that any Lie $p$-subalgebra of free Lie $p$-algebra is free [12]).

We recall now several definitions and results about $L_{p}\langle X\rangle$.

A linear basis of $L_{p}\langle X\rangle$ are all $p$-proper words [2] which are formed from symbols $\left\{x_{1}, x_{2}, \ldots, x_{n}\right\}$. If $L\langle X\rangle$ denotes a free Lie algebra free generated by the set $X$, then the proper words of $L_{p}\langle X\rangle$ are formal $p^{k}$-degrees of proper words of $L\langle X\rangle$.

We shall use the ordinary concept of degree of element from $L_{p}\langle X\rangle$; for example if $f=x_{\alpha} x_{\beta}+x_{\gamma}^{p}$, then $\operatorname{deg} f=p$. We assume that $\operatorname{deg} 0=0$.

Suppose $f \in L_{p}\langle X\rangle, f=\sum_{i} \alpha_{i} q_{i}$, where $q_{i}$ are $p$-proper words. Such a record of $f$ is called a right form of $f$. An element $f^{\prime}=\sum_{i \in I} \alpha_{i} q_{i}$ where degi $=\operatorname{deg} f$ and degi $<\operatorname{deg} f$ if $i \notin I$ is called a major part of $f$. Let $\tilde{f}$ denote the major member of $f \in L_{p}\langle X\rangle$ defined as a lexicographically major word among $q_{i}, i \in I$. About these concepts see [2]. 
A subset $Y \subset L_{p}\langle X\rangle$ is called $p$-reduced [3] if for any $f \in Y$ his major part $f^{\prime}$ does not belong to Lie $p$-subalgebra of $f \in L_{p}\langle X\rangle$ generated by major parts of all elements from $Y \backslash\{f\}$. We assume that the empty set is $p$-reduced. Let $Y=\left\{y_{1}, y_{2}, \ldots, y_{m}\right\} \subset L_{p}\langle X\rangle$ be a finite set. A map $t: Y \rightarrow L_{p}\langle X\rangle$ is called elementary if for some $j$

$$
\begin{gathered}
t\left(y_{i}\right)=y_{i}, \text { if } i \neq j \\
t\left(y_{j}\right)=\alpha y_{j}+\varphi_{j}\left(y_{1}, y_{2}, \ldots, y_{m}\right), \text { where } y_{j} \text { is missed; }
\end{gathered}
$$

here $\alpha \in k, \alpha \neq 0$ and $\varphi_{j}$ are polynomials i.e. elements of free Lie $p$-algebra with $m$ free generators.

Let $Y^{\prime}$ denotes a set of major parts of elements from $Y^{\prime}$ with respect to standard ordering considered in the beginning this paper. Put

$$
l(Y)=\sum_{i} \operatorname{deg}\left(y_{i}\right)
$$

As we have already noted degy $y_{i}$ is the length of longest word in $y_{i}$ and $\operatorname{deg} 0=0$.

Lemma 1. Let $\left\{y_{1}, y_{2}, \ldots, y_{m}\right\}$ be a finite set of generators of $L_{p}\langle X\rangle$. Then it exist $l(Y)-n$ (here $n$ is a number of free generators of $L_{p}\langle X\rangle$ ) elementary maps which translate $Y$ onto a set of generators of $L_{p}\langle X\rangle$ with degrees (regarding to $X$ ) less or equal one.

Remark 1. This lemma was proved in [2] for Lie algebras; we prove our lemma in the same manner.

Proof. We may assume that $\mathrm{Y}$ contains at least one element; otherwise there is nothing to prove. Let us prove that $Y^{\prime}$ is not $p$-independent. Since $Y$ generates $L_{p}\langle X\rangle$ we must have

$$
x_{i}=\sum_{i=1}^{m} \alpha_{i j} y_{j}+f_{i}\left(y_{1}, y_{2}, \ldots, y_{m}\right),
$$

where $f_{i}$ does not contain elements of degree one. Assume all $f_{i}$ are zero:

$$
x_{i}=\sum_{i=1}^{m} \alpha_{i j} y_{j}, i=1,2, \ldots, n .
$$

Let us compare elements with highest degrees in (3). Assume that there exists $j_{0}$ such that $\operatorname{deg}\left(y_{j_{0}}\right)>1, \alpha_{i_{0} j_{0}} \neq 0$. Then

$$
\left(x_{i_{0}}\right)^{\prime}=x_{i_{0}}=\left(\sum_{j=1}^{m} \alpha_{i_{0}} y_{j}\right)^{\prime} .
$$

Let us denote $J=\left\{j \mid \operatorname{deg}\left(y_{j}\right)=\operatorname{deg}\left(y_{j_{0}}\right)\right\}$, then from (4) follows

$$
\sum_{j \in J} \alpha_{i_{0}} y_{j}^{\prime}=0
$$

i.e. $Y^{\prime}$ is not $p$-independent because otherwise we would have $\operatorname{deg}\left(x_{0}\right)>1$.

On the other hand, if in (2) we have that if $(\forall i, j)\left(\alpha_{i j} \neq 0\right.$ implies $\left.\operatorname{deg}\left(y_{i}\right)=0\right)$, then from (3) it follows

$$
x_{i}^{\prime}=x_{i}=\sum_{j \in J_{i}} \alpha_{i j} y_{j}^{\prime}, i=1,2, \ldots, n .
$$


Any element from $Y^{\prime}$ is generated by elements $x_{i}$, therefore according to (5) all elements from $Y^{\prime}$, and among those with the degrees greater one, are generated by elements $y_{j}^{\prime}, j \in \bigcup_{i} J_{i}$, i.e. $Y^{\prime}$ is not $p$-independent.

Now suppose that in (2) $f_{i_{0}}\left(y_{1}, y_{2}, \ldots, y_{m}\right) \neq 0$ for some $i_{0}$. If $f_{i_{0}}\left(y_{1}^{\prime}, y_{2}^{\prime}, \ldots, y_{m}^{\prime}\right)=0$ then $Y^{\prime}$ is not $p$-independent. Now suppose $f_{i_{0}}\left(y_{1}^{\prime}, y_{2}^{\prime}, \ldots, y_{m}^{\prime}\right) \neq 0$. Let us write it as

$$
f_{i_{0}}\left(y_{1}^{\prime}, y_{2}^{\prime}, \ldots, y_{m}^{\prime}\right)=\sum_{j=1}^{s} h_{j}\left(x_{1}, x_{2}, \ldots, x_{n}\right),
$$

where $h_{j}$ is a homogeneous component of degree $d_{i}$ of $f_{i_{0}}\left(y_{1}^{\prime}, y_{2}^{\prime}, \ldots, y_{m}^{\prime}\right), d_{1}<d_{2}<\ldots<d_{s}$. Because $y_{i}^{\prime}$ are homogeneous, each polynomial $h_{j}\left(x_{1}, x_{2}, \ldots, x_{n}\right)$ must be a polynomial of arguments $y_{1}^{\prime}, y_{2}^{\prime}, \ldots, y_{m}^{\prime}$ :

$$
h_{j}\left(x_{1}, x_{2}, \ldots, x_{n}\right)=q_{j}\left(y_{1}^{\prime}, y_{2}^{\prime}, \ldots, y_{m}^{\prime}\right) .
$$

Therefore from (6) follows

$$
f_{i_{0}}\left(y_{1}^{\prime}, y_{2}^{\prime}, \ldots, y_{m}^{\prime}\right)=\sum j=1^{s} q_{j}\left(y_{1}^{\prime}, y_{2}^{\prime}, \ldots, y_{m}^{\prime}\right),
$$

where $q_{j}\left(y_{1}^{\prime}, y_{2}^{\prime}, \ldots, y_{m}^{\prime}\right) \neq 0, j=1,2, \ldots, s$, otherwise $Y^{\prime}$ would have not been $p$-independent; in particular $q_{s}\left(y_{1}^{\prime}, y_{2}^{\prime}, \ldots, y_{m}^{\prime}\right)=0$. Consequently

$$
\left(f_{i_{0}}\left(y_{1}^{\prime}, y_{2}^{\prime}, \ldots, y_{m}^{\prime}\right)\right)^{\prime}=f_{i_{0}}\left(y_{1}^{\prime}, y_{2}^{\prime}, \ldots, y_{m}^{\prime}\right)=q_{s}\left(y_{1}^{\prime}, y_{2}^{\prime}, \ldots, y_{m}^{\prime}\right) .
$$

From (2) follows

$$
x_{i}=x_{i}^{\prime}=\left(\sum_{j=1}^{s} \alpha_{i_{0} j} y_{j}+f_{i_{0}}\left(y_{1}, y_{2}, \ldots, y_{m}\right)\right)^{\prime} .
$$

Two cases are now possible.

1. $f_{i_{0}}\left(y_{1}^{\prime}, y_{2}^{\prime}, \ldots, y_{m}^{\prime}\right)=q_{s}\left(y_{1}^{\prime}, y_{2}^{\prime}, \ldots, y_{m}^{\prime}\right)$ is contained in the major part of $\sum_{j=1}^{m} \alpha_{i_{0} j} y_{j}$; then because the degree of $x_{i}$ is one, for some $J \subset\{1,2, \ldots, m\}$ we must have (see $(7)$ ):

$$
\sum_{j \in J}^{m} \alpha_{i_{0} j} y_{j}+q_{s}\left(y_{1}^{\prime}, y_{2}^{\prime}, \ldots, y_{m}^{\prime}\right)=0 \text {. }
$$

i.e. $Y^{\prime}$ is not $p$-independent.

2. $f_{i_{0}}\left(y_{1}^{\prime}, y_{2}^{\prime}, \ldots, y_{m}^{\prime}\right)=q_{s}\left(y_{1}^{\prime}, y_{2}^{\prime}, \ldots, y_{m}^{\prime}\right)$ is not contained in the major part of $\sum_{j=1}^{m} \alpha_{i_{0} j} y_{j}$; then $\sum_{j=1}^{m} \alpha_{i_{0} j} y_{j}$ contains letters $y_{j}$ such that their degree are greater than $d_{s}$ and consequently, greater than one. Let $y_{j}, j \in J$ be all $y_{j}$ from $\sum_{j=1}^{m} \alpha_{i_{0}} y_{j}$ (of course with nonzero coefficients) having the highest degree; then

$$
\sum_{j=1}^{m} \alpha_{i_{0} j} y_{j}=0
$$

because $\operatorname{deg}\left(x_{i_{0}}=1\right.$ (see $(7)$, i.e. $Y^{\prime}$ is not $p$-independent. So we have considered all cases and have proved that $Y^{\prime}$ is not $p$-independent. In ([2], Lemma 2) was proved that a $p$-reduced subset of free Lie $p$-algebra is $p$-independent. From the above lemma follows, because $Y^{\prime}$ is not $p$-independent, 
that $Y^{\prime}$ is not $p$-reduced. Therefore there exists an element $y_{j_{0}}^{\prime} \in\left(Y^{\prime}\right)^{\prime}=Y^{\prime}$ such that $y_{j_{0}}^{\prime}$ is contained in a $p$-subalgebra of $L_{p}\langle X\rangle$ generated by a set $Y^{\prime} \backslash\left\{y_{j_{0}}^{\prime}\right\}$ i.e.

$$
y_{j_{0}}^{\prime}=q\left(y_{1}^{\prime}, \ldots, \hat{y}^{\prime}, \ldots, y_{m}^{\prime}\right),
$$

where $q\left(y_{1}^{\prime}, \ldots, \hat{y}^{\prime}, \ldots, y_{m}^{\prime}\right)$ does not contain $y_{j_{0}}^{\prime}$. Consequently a map

$$
y_{i}^{(1)}=y_{i}, i \neq j_{0}, y_{j_{0}}^{(1)}=y_{j_{0}}-q\left(y_{1}^{\prime}, \ldots, \hat{y}^{\prime}, \ldots, y_{m}^{\prime}\right)
$$

reduces $l(Y)$ (see $(1))$. Indeed from $(8)$ follows that $q\left(y_{1}, \ldots, \hat{y}^{\prime}, \ldots, y_{m}\right) \neq 0$ and therefore

$$
y_{j_{0}}=q\left(y_{1}^{\prime}, \ldots, \hat{y}^{\prime}, \ldots, y_{m}^{\prime}\right)=q\left(y_{1}, \ldots, \hat{y}, \ldots, y_{m}\right)^{\prime},
$$

i.e. (9) reduces $l(Y)$.

Lemma 2. Assume a set $Z=\left\{z_{1}, z_{2}, \ldots, z_{m}\right\}$ generates $L\langle X\rangle$ and $\operatorname{deg}_{X} z_{i} \leq 1$. If $\left\{z_{1}, z_{2}, \ldots, z_{m_{0}}\right\}$ is a maximal linearly independent subset of $Z$, then there exist $m-m_{0}$ elementary maps which transform $Z$ onto the set $\left\{z_{1}, z_{2}, \ldots, z_{m_{0}}, 0, \ldots 0\right\}$.

Proof. Let $z_{j}=\sum_{j=1}^{m_{0}} \alpha_{i j} z_{j}, i=m_{0}+1, m_{0}+2, \ldots, m$. Then it is clear that the sought maps are

$$
\begin{gathered}
\tilde{z}_{i}=z_{i}, i=1,2, \ldots, m \\
\tilde{z}_{i}=z_{i}-\sum_{j=1}^{m_{0}} \alpha_{i j} z_{j}, i=m_{0}+1, m_{0}+2, \ldots, m .
\end{gathered}
$$

Recall that $F=k\langle X\rangle$ is the free associative algebra over set $X=\left\{x-1, x_{2}, \ldots, x_{n}\right\}$ without iden- tity (of course $X \subset F$ ). For $a \in F$, let $\langle a\rangle$ be an ideal of $F$ generated by $a$. It is clear that $a \in\langle a\rangle$. Let $\bar{a}$ be the major word of $a$.

Lemma 3. If $a, b \in k\langle X\rangle$ and $\langle a\rangle=\langle b\rangle$, then $a$ and $b$ are linearly dependent.

Proof. If either $\langle a\rangle$ or $\langle b\rangle$ are zero, our proposition of course is valid. So we may assume that $a, b \neq 0$. From [1] follows that if $x \in\langle a\rangle$, then $\bar{a}$ is a subword of $\bar{x}$. Therefore $\bar{a}$ is a subword of $\bar{b}$ and, conversely, $\bar{b}$ is a subword of $\bar{a}$ and consequently $\bar{a}=\bar{b}$. Suppose

$$
a=\alpha \bar{a}+\ldots, b=\beta \bar{b} ; \alpha, \beta \in k, \alpha, \beta \neq 0 .
$$

Consider the element $c=a-\frac{\alpha}{\beta} b \in\langle a\rangle=\langle b\rangle$. If $c \neq 0$, then $\bar{c}$ is less then $\bar{a}$. On the other hand, $\bar{a}$ is a subword of $\bar{c}$ - contradiction, so $c=0$.

Corollary 1. Let $F_{1}=k\langle X\rangle_{1}$ be a free associative algebra with identity which is freely generated by $X$. Suppose $a, b \in F_{1}$ and $\langle a\rangle=\langle=b\rangle$. Then $a$ and $b$ are linearly dependent.

Proof. This is clear since $\langle a\rangle$ is an ideal in $F=k\langle X\rangle$ if and only if $\langle a\rangle$ is the ideal in $F_{1}=k\langle X\rangle_{1}$.

Let $\langle a\rangle$ denote an ideal of $L_{p}\langle X\rangle$ generated by $a \in L_{p}\langle X\rangle$ (we assume $a \in\langle a\rangle$ ) and let $\bar{a}$ be the major word of $a$.

Corollary 2. Let $\langle a\rangle=\langle b\rangle \subseteq L_{p}\langle X\rangle$. Then $a$ and $b$ are linearly dependent.

Proof. As is well known, $u\left(L_{p}(X)\right)=k\langle X\rangle_{1}=F_{1}$ (here $u\left(L_{p}(X)\right)$ is a restricted universal enveloping algebra of $\left.L_{p}(X)\right)$. Let $\langle a\rangle$ and $\langle b\rangle$ be the ideals in $F_{1}=k\langle X\rangle_{1}$, generated, respectively by $a$ and $b$. It is clear that

$$
\langle a\rangle_{1}=\langle b\rangle_{1} \subseteq F\langle X\rangle
$$


then according to Lemma 3 the elements $a$ and $b$ are linearly dependent.

Definition. An element $a \in L_{p}\langle X\rangle$ is primitive if there exist a set $Y$ of free generators of $L_{p}\langle X\rangle$ such that $a \in Y$.

Theorem. $L_{p}\langle X\rangle /\langle a\rangle$ is free if and only if $a$ is primitive in $L_{p}\langle X\rangle$.

Proof. It is clear that if $a$ is primitive then $L_{p}\langle X\rangle /\langle a\rangle$. Suppose that $L_{p}\langle X\rangle /\langle a\rangle$ is free and let us prove that $a$ is primitive.

Let us denote $\bar{L}=L_{p}\langle X\rangle /\langle a\rangle$. It is clear that $\operatorname{dim} \bar{L} / \bar{L}^{2} \geq n-1$. Indeed

$$
\bar{L} / \bar{L}^{2}=\left(L_{p}\langle X\rangle /\langle a\rangle\right) /\left(L_{p}\langle X\rangle /\langle a\rangle\right)^{2} \cong L_{p}\langle X\rangle /\left(L_{p}\langle X\rangle^{2}+\langle a\rangle\right)
$$

but last term as $k$-vector space is isomorphic to $\left(k x_{1}+k x_{2}+\ldots+k x_{n}+\langle a\rangle\right) /\langle a\rangle$, which implies that $\operatorname{dim}\left(\bar{L} / \bar{L}^{2}\right) \geq n-1$.

On the other hand, $L_{p}\langle X\rangle$ is generalized nilpotent, i.e. intersection all its degrees is zero. According to [5] all generalized nilpotent algebras are Hopf type, i.e. they are not isomorphic to their proper factor- algebras. Consequently,

$$
\operatorname{rank} \bar{L}=\operatorname{rank}\left(L_{p}\langle X\rangle /\langle a\rangle\right) \leq n-1 .
$$

However, if $\operatorname{rank}(\bar{L}) \leq n-1$, then $\operatorname{rank}\left(\bar{L} / \bar{L}^{2}\right)<n-1$; so $\operatorname{rank}(\bar{L})=n-1$ and there exist a set of free generators $Y=\left\{y_{1}, y_{2}, \ldots, y_{n-1}\right\}$ for $\bar{L}$. The set $X=\left\{x_{1}, x_{2}, \ldots, x_{n}\right\}$ generates $\bar{L}$ and by to Lemma 1 there exist elementary maps which transform $\bar{X}$ in a set of generators $Z=\left\{z_{1}, z_{2}, \ldots, z_{r}\right\}$ of $\bar{L}$ such that degrees of $z_{i}, i=1,2, \ldots, r$ with respect $Y$ are not greater than one. By lemma 2 there exist elementary maps which transform $Z=\left\{z_{1}, z_{2}, \ldots, z_{r}\right\}$ onto $\left\{z_{1}, z_{2}, \ldots, z_{r_{0}}, 0, \ldots, 0\right\}$, where $\left\{z_{1}, z_{2}, \ldots, z_{r_{0}}\right\}$ is a maximal linearly independent set in $L$. It is clear that $r_{0}=n-1$ and number of zeros in $\left\{z_{1}, z_{2}, \ldots, z_{r_{0}}, 0, \ldots, 0\right\}$ is one, therefore some elementary maps transform $\left\{z_{1}, z_{2}, \ldots, z_{r_{0}}, 0\right\}$ on $\left\{y_{1}, y_{2}, \ldots, y_{r_{0}}, 0\right\}$ (if this set contains only zero then $n=1$ ). Therefore we may assume that there exist elementary maps $\varphi_{1}, \varphi_{2}, \ldots, \varphi_{s}$ which transform $\bar{X}=\left\{\bar{x}_{1}, \bar{x}_{2}, \ldots, \bar{x}_{n}\right\}$ onto $\left\{y_{1}, y_{2}, \ldots, y_{r_{0}}, 0\right\}$. The elements $X=\left\{x_{1}, x_{2}, \ldots, x_{n}\right\}$ are preimages of $\bar{X}=\left\{\bar{x}_{1}, \bar{x}_{2}, \ldots, \bar{x}_{n}\right\}$. The maps $\varphi_{1}, \varphi_{2}, \ldots, \varphi_{s}$ transform $X$ on a set $\left\{t_{1}, t_{2}, \ldots, t_{n}\right\}$ of free generators of $L_{p}\langle X\rangle$. Let us consider a projection $\pi: L_{p}\langle X\rangle \rightarrow L_{p}\langle X\rangle /\langle a\rangle$. From a commutative diagram below it is clear that $\pi\left(t_{n}\right)=0$ :

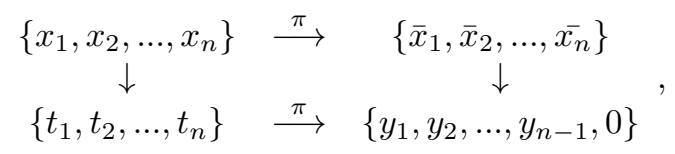

where vertical maps are equal to composition $\varphi$ of the maps $\varphi_{1}, \varphi_{2}, \ldots, \varphi_{s}$. So $t_{n} \in\langle a\rangle$, i.e. $\left\langle t_{n}\right\rangle \in$ $\langle a\rangle$. In fact, $\left\langle t_{n}\right\rangle=\langle a\rangle$. Indeed let us consider an algebra $L_{p}\langle X\rangle /\left\langle t_{n}\right\rangle$. It is free. As $\left\langle t_{n}\right\rangle \in\langle a\rangle$ so

$$
\left(L_{p}\langle X\rangle /\left\langle t_{n}\right\rangle\right) /\left(\langle a\rangle /\left\langle t_{n}\right\rangle\right) \cong L_{p}\langle X\rangle /\langle a\rangle .
$$

As $L_{p}\langle X\rangle /\left\langle t_{n}\right\rangle$ and $L_{p}\langle X\rangle /\langle a\rangle$ are free Lie $p$-algebras with $n-1$ generators, and free Lie $p$-algebras are Hopf type algebras we must have $\langle a\rangle /\left\langle t_{n}\right\rangle=0$, i.e. $\langle a\rangle=\left\langle t_{n}\right\rangle$. Then from Corollary 2 follows that $a=\alpha t_{n}$ for some $\alpha \in k$, i.e. $a$ is primitive.

Remark 2. We assume that the other results from [2] can be proved in the same way.

Remark 3. J. P. Serre has proved the following theorem ([11], [10]): 
Theorem (Serre). Let $R$ be a commutative ring and let $G$ be a group having no $R$-torsion. If $H$ is a subgroup of finite index in $G$, then $c d_{R} G=c d_{R} H$.

We assume that an analogous statement about Lie p-algebras is also valid: let $L$ be a Lie $p$ algebra such that restricted universal algebras of all finite Lie $p$-subalgebras of $L$ are semisimple. If $H$ is a Lie $p$-subalgebra of finite index in $L$, then $c d_{L} G=c d_{R} H$.

Acknowledgment. This work is partially supported by GNSF grant FR/307/5-113/13.

\section{References}

[1] L. A. Bokut, Embedddings in prime associative algebras, Algebra i Logica, 15(2) (1976), 117 142 (in Russ.)

[2] G. P. Kukin, Primitive elements of free Lie algebras, Algebra i Logica, 9(4) (1970), 458-472 (in Russ.).

[3] G. P. Kukin, On subalgebras of free Lie p-algebras, Algebra i Logica 11(5) (1972) 535-550 (in Russ.).

[4] W. Magnus, Uber diskontinuierliche Gruppen mit einer definierden Relation (Der Freiheitssatz), J. Reine Angew. Math. 163 (1930), 141-165.

[5] A. I. Malcev, On algebras with identity relations, Mat. Sborn. 26/1 (1950), 19-33 (Russ.).

[6] A. A. Mikhalev, U. U. Umirbaev and J.-T. Yu, Automorphic orbits in free nonassociative algebras. J. Algebra, 243 (2001), 198-223.

[7] A. A. Mikhalev, V. Shpilrain and U. U. Umirbaev, On isomorphism of Lie algebras with one defining relations. Int. J. Algebra Comput. 14(3) (2004), 389-394.

[8] G. Rakviashvili, Combinatorial aspects of free associative algebras and cohomologies of Lie palgebras with one defining relation, Journal of Mathematical Sciences 160(6) (2009), 822-832.

[9] G. Rakviashvili, Primitive elements of free Lie p-algebras, Bull. of the Georgian Academy of Sciences, 8(2) (2014), 14-18.

[10] J.-P. Serre, Cohomologie des groups discrets Ann. Math. Studies 70 (1971), 77-169

[11] R. G. Swan, Groups of cohomological dimension one, J. of Algebra 12 (1969), 585-604.

[12] E. Witt, Die Unterringe freien Lieschen Ringe. Math. Z. 64(2) (1956), 195-216. 